\title{
Multimodal Intraoperative Neurophysiological Monitoring in Spinal Cord Surgery
}

\author{
Emine TASKIRAN ${ }^{1}$, Sema BRANDMEIER ${ }^{2}$, Erdinc OZEK ${ }^{3}$, Ramazan SARI ${ }^{3}$, Fatihhan BOLUKBASI ${ }^{3}$, \\ Ilhan ELMACI ${ }^{3}$

\begin{abstract}
${ }^{1}$ Istanbul University, Cerrahpasa Medical Faculty, Department of Neurosurgery, Intraoperative Neurophysiology, Istanbul, Turkey ${ }^{2}$ Istanbul University, Experimental Medicine Institute, Neuroscience Department, Electroneurophysiology, Istanbul, Turkey ${ }^{3}$ Memorial Health Group, Neurosurgery Clinics, Istanbul, Turkey
\end{abstract}

This study was presented at $31^{\text {st }}$ National Clinical Neurophysiology EEG-EMG Congress at 8-12 April, 2015 in Antalya, Turkey and $5^{\text {th }}$ International Society of Intraoperative Neurophysiology at 9-14 November, 2015 in Brazil.

\section{ABSTRACT}

AIM: Intraoperative neurophysiological monitoring (IONM) monitors the functional integrity of critical neural structures by electrophysiological methods during surgery. Multimodality combines different neurophysiological methods to maximize diagnostic efficacy and provide a safety margin to improve the outcomes of spinal surgery. Our aim was to share our intraoperative monitoring experiences with patients who underwent surgery because of spinal cord pathologies between September 2013 and January 2015.

MATERIAL and METHODS: We had twenty-six cases. Location of the lesions, surgery, neurological findings, and electrophysiological findings intraoperatively and postoperatively were documented.

RESULTS: The combination of motor evoked potential (MEP), somatosensorial evoked potential (SSEP), free-run and trigger electromyography (EMG) were performed according to lesion localization. MEPs plus SSEPs were run in 23 patients and MEPs with triggered EMG were performed in 4 patients. In only one patient, optimal recording could not be elicited because of technical problems. MEP and SSEP changes were recorded in 12 and 3 patients respectively. Postoperative neurological deficits were observed in 2 patients. Deficits were transient in one case and permanent in the other. While baseline MEP responses were either absent or low amplitude ( $<50$ microvolt) in 7 patients, following resection they were either visible or increased in amplitude. Surgery was ended in one patient with C7-T2 intramedullary tumour after the right distal MEP response disappeared.

CONCLUSION: Multimodal IONM is an important method to monitor the neural structures under risk in spine surgery and to keep the surgery within safety limits, especially for intramedullary spinal cord lesion surgery.

KEYWORDS: Spinal cord surgery, Evoked potentials, Multimodality, Intraoperative monitoring

\section{INTRODUCTION}

S pinal surgeries carry a very high risk of injury to the spinal cord, spinal nerves and/or blood vessels. Neurological deficits, such as paralysis, muscle weakness, pain, bladder/bowel disturbances and sexual dysfunction, which emerge after these surgeries can seriously affect basic human functions and increase morbidity $(1,3,11,14)$.
Intraoperative neurophysiological monitoring (IONM) is the use of electrophysiological methods to monitor the functional integrity of critical neural structures during surgery. IONM can predict an increased risk of injury during surgery based on electrophysiological changes. The most commonly used electrophysiological modalities are evoked potentials (Somatosensory Evoked Potentials - SSEP and Motor Evoked Potentials - MEP) and electromyography (EMG) in spinal cord 
surgery. Multimodality is the combination of different modalities such as SSEP and MEP or MEP, SSEP and EMG, etc. This kind of combination maximizes the reliability of findings related to the neural structures under risk, which in turns keeps surgical maneuvers within safe limits and prevents iatrogenic damage. In this study, we aimed to share our intraoperative monitoring experiences with 26 patients who undertook surgery between September 2013 and January 2015 because of spinal cord pathologies.

\section{MATERIAL and METHODS}

\section{Patient Population}

Multimodal IONM was performed during neurosurgery in 26 cases, including 10 children and 16 adults between September 2013 and January 2015. These patients consisted of 14 males (53\%) and 12 females as sex distribution. The mean age of the patients was 33.2 years (median 42 years, range 4-71 years).

\section{Monitoring}

Monitoring was performed using the Medtronic NIM-Eclipse spinal system version 3.5.353 for IONM. MEPs were recorded to assess the anterior motor pathways while SEPs were obtained to monitor the sensory pathways in the dorsal columns. Additionally, free-run electromyography (fEMG) and direct nerve root/rootlet stimulation were performed. IONM was performed by a single technician and 2 neurophysiologists (E.T, S.B). All surgical procedures were followed on the visual screen in the operating room during surgery.

Total intravenous anesthesia (TIVA) consisting of propofol and analgesic drugs (remifentanil) was used in all cases. We avoided using volathile anesthetics because they reduce the tcMEP amplitude significantly more than propofol. In particular, this effect was considerably distinct in the patients who had preexisting neurologic deficiencies $(10,19,23)$. A short half-life muscle relaxant was used only during tracheal intubation procedure. The elimination of the muscle relaxant was conformed by performing TOF (train of four).

Monitoring was started immediately after anesthesia induction and continued until the termination of the surgical procedure, as recommended by the American Clinical Neurophysiology Society (2009) (15).

The selection of muscles to be monitored was based on level of the lesion. Disposable corkscrew (CS) electrodes were used for stimulation of MEPs and also for recording of cortical SSEPs.

We used $13 \mathrm{~mm}$ stainless still needle electrodes (Medtronic ${ }^{\circledR}$ ) to record muscles' responses for MEPs and EMG and to stimulate peripheral nerves for SSEP.

MEP stimulating electrodes were placed at C1/C2 for the lower extremities and $\mathrm{C} 3 / \mathrm{C} 4$ for the upper extremities, according to international 10-20 system for EEG. MEP responses were recorded from the appropriate muscles the lower and upper extremities depending upon the level of the lesion. Double train stimulation was used for TcMEP. Each train consisted of 5 pulses of $0.5 \mathrm{~ms}$ duration with interstimulus intervals (ISI) of $3 \mathrm{~ms}$. Stimulation intensities ranged between 200-400 V. Stimulus duration was $50 \mathrm{~ms}$.

The SSEPs in upper extremities were obtained by stimulating median or ulnar nerves and recording cortically with an electrode montage of $\mathrm{C}^{\prime}$ '-FPz/C4'-FPz, while those of the lower extremities' were obtained by stimulating the tibial nerves recording from cortical $\mathrm{Cz}$ '-FPz. All stimulations were performed bilaterally with interleaving stimulation for upper and lower extremities.

SSEP recordings were set up with a sensitivity of $0.5 \mu \mathrm{V} /$ $\mathrm{mm}$ and a sweep speed of $100 \mathrm{msec}$; filter settings were set to $100-300 \mathrm{~Hz}$; the stimulus frequency was $1.7 \mathrm{~Hz}$, and the stimulation duration was $500 \mu \mathrm{s}$ for the tibial nerve and 300 $\mu$ s for the median/ulnar nerve. The mean stimulus intensity was 20 and $30 \mathrm{~mA}$ for the median/ulnar and tibial nerves, respectively.

\section{RESULTS}

The cases consisted of 14 males and 12 females, ranging in age from 4 to 71 (with a mean age of 33.2 years). The distribution of the types of surgeries in these series is shown in Figure 1. The most common surgery was intradural intramedullary spinal cord tumour surgery (38\%).

We performed MEP, SSEPs and free running EMG in all patients. In addition, triggered EMG was applied in 4 of the cases. We could not elicit reliable recordings in only one patient, and that was due to technical problems. The most common monitored muscles for MEP were the abductor pollicis brevis (APB), abductor hallucis brevis (AHB), anterior tibialis (AT), and biceps brachii (BB). We observed MEP changes in 12 patients and SSEP changes in 3 patients.

Changes in MEP responses that we observed electrophysiologically consisted of either increased responses, or responses that remained at baseline, or the appearance of MEP responses that were not present at the baseline following tumour resection. The surgery was ended in one patient with C7-T2 intramedullary tumour because the right distal MEP response disappeared. Fortunately, this patient had a transient postoperative neurological deficit that recovered in a few hours.

SSEP changes that we observed in 3 patients were that the right SEP latency increased more than $10 \%$ in one. This patient showed paresthesia in the right upper extremity postoperatively. The other SEP change was that amplitudes decreased more than $50 \%$. This change was seen in another patient who had intradural intramedullary tumour at C3-6. The surgeon was warned as soon as that was seen. The surgery process was halted for a few minutes. After 15 minutes the response came back.

Triggered EMG was used to help the surgeon to identify functional nerve roots. Direct spinal stimulation was performed by the same neurosurgeon. We used monopolar and/or bipolar probe to stimulate and identify objectively nerve roots and non-nervous tissue like the terminal filum for tethered cord surgery. We preferred not to increase the stimulation intensity 


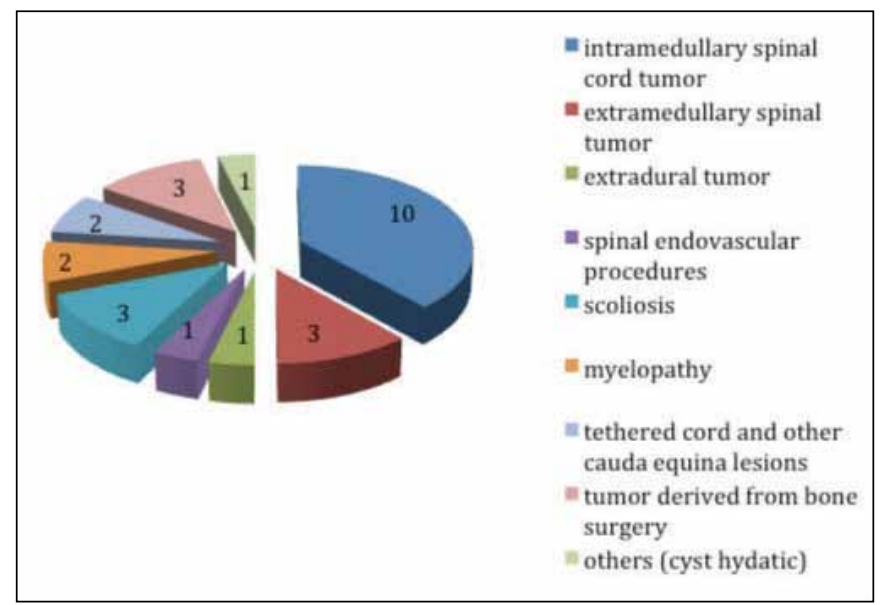

Figure 1: The distribution of the cases based on diagnosis.

more than $5 \mathrm{~mA}$ in order to prevent the current spread for monopolar stimulation. In addition, we paid attention to the stimulation of little rootlets of S3-4, which might cling the to the filum. In this situation, we should keep in mind that while the surgeon might point out to the absence of nerves in this kind of operative region, we may still get a response.

Postoperatively, we saw neurological deficits in two patients, one which had transient deficits and the other with permanent deficits. This latter already had paraparesia before surgery. She had intradural intramedullary tumour at the Thoracic 2 level and we could not record any SSEP and MEPs responses at the beginning, during and at the end of surgery in this patient.

\section{DISCUSSION}

It has been shown that the sensitivity and specificity is high for multimodal intraoperative monitoring with SSEPs and MEPs as compared to using only one modality $(2,7,13)$. Feng et al., retrospectively reviewed 176 patients who underwent spinal surgery and were monitored with MEP or SSEP and MEP plus SSEP. Just using SSEP had specificity (92\%) but no sensitivity. Therefore it could detect about half the cases of nerve injury. Just MEP monitoring had a high sensitivity (92\%) and specificity (95\%). Combining SSEP and MEP monitoring revealed the highest performance in terms of both sensitivity $(93 \%)$ and specificity (99\%)(2).

IONM can be used even in younger ages $(17,22)$. The immature myelinated corticospinal fibers in these patients may desynchronize the conveyance of action potentials to the motor neurons. This often results in failure to elicit muscle MEPs. Even so, responses can always be obtained when no halogenated agents are administered and patients do not have severe neurological deficits $(8,23)$. And also, using double train stimulation compensates the relative hyperpolarisation of motor neurons resulting in reliable MEP responses in this situation $(5,6)$. It is only a worthy of notice feature that EEG cup electrode usage is recommended for young children. The CS electrodes could penetrate the fontanel during placement in whom the fontanel still exists. In our series, we used the double train stimulation technique to elicit MEPs in all patients and the stimulation intensity and duration were the same in both children and adults.

The most frequently carried out surgery in our study was for intradural intramedullary spinal cord tumour (IMSCT) (38\%). Surgically removing this kind of tumour is important, because a great majority of them are histologically benign. Furthermore, if they can be removed totally, especially for some tumours such as ependymoma, resection alone may provide a cure (16). Permanent neurological deficiency occurred in only one patient who already had deficits before surgery. The neurological deficit in the other patients recovered in a few hours.

Monitoring of TcMEPs solely gives information about the anterolateral columns of the spinal cord, while the monitoring of SSEPs provides information from the posterior columnlemniscal system. SSEP and MEP methods are complementary methods to monitor spinal cord integrity. Therefore, we performed the combination of MEP+SSEP, or MEP+SSEPs with the addition of free run and/or triggered EMG according to lesion level.

The selection of appropriate muscles to record is an important issue in the monitoring of MEPs. To choose optimal recording muscles in the patients who already have severe paresis means modifications according paretic muscles must be made to avoid "non-monitorable" cases. On the other hand, the small hand muscle like APB is one of the optimal muscles to monitor the cortical spinal tract (CT) for the upper extremities. Other upper extremity muscles are the long forearm flexors or the forearm extensors (20). These muscles have a rich CT innervation, making them suitable to monitor the functional integrity of the CT.

Abductor hallucis brevis is the optimal muscle for the lower extremities for the same reason. In the experimental studies, the highest amplitude of the excitatory postsynaptic potential (EPSP) in the alfa motoneuron pools for the lower extremities was found in the small and long flexors of the foot following CT stimulation (4). Based on these previous studies, our standard electrodes recording sites for MEPs were AHB and AT for the lower extremity recordings and APB for the upper extremity recordings. And we preferred to choose more than one muscle, because we could not record the $D$ wave, which was our limitation.

EMG monitoring consists of two main techniques: free run EMG and triggered EMG. Both techniques monitor muscles supplied by specific nerve roots in the upper and lower extremities. EMG recordings are obtained by inserting needle electrodes into the muscles. They allow recording of the muscle action potentials generated by nerve roots at risk during surgery (12). We used the triggered EMG technique to assist the accurate placement of pedicle screws in scoliosis, and to also identify functional nerve tissue in tethered cord and cauda equina lesion surgeries.

We had some limitations in this study. One of them is related to the $D$ wave, which is recorded directly from the 
spinal cord. D wave is an essential complementary method to monitor CT functional integrity as the "gold standard". D waves have straightforward warning criteria despite limited indications, while muscle MEPs have many indications with unsettled warning criteria. D waves are compound volleys of corticospinal axon action potentials. They are non-synaptic, resistant to anesthesia and neuromuscular blockade (NMB). Their amplitudes are proportional to the number of conducting axons. They show $<10 \%$ trial-to-trial amplitude variability and they are therefore accepted to be stable. On the other hand, Muscle MEPs are compound motor unit potentials, generated by lower motor neuron (LMN) temporal summation of multiple corticospinal EPSP. They are polysynaptic, resistant to anesthesia and NMB. They are non-linear. Although the presence of disproportionately large amplitude decrements, it can be small reductions of conducting corticospinal axons or LMN excitability. They are unstable and show the variability of amplitude, morphology, and threshold. Although muscle MEP disappearance is always a major criterion, as it is a strong predictor of early postoperative weakness when irreversible in the IMSCT, the D wave amplitude warning criterion for this surgery is a reduction of $50 \%$, predicting long term motor deficiency (9). Unfortunately, we could not add this monitoring to our set up, due to the cost of the recording electrodes and our limited experience with this modality. This was a limitation in monitoring our cases. On the other hand, Nuwer et al. concluded that multimodal IONM with SSEPs with MEPs is effective to predict an increased risk of the worst outcomes of paraparesis, paraplegia, and tetraplegia in spinal surgery (13). According to this paper, monitoring additional muscles for MEPs in combination with SSEPs should be considered for such cases.

Another limitation in this study was not using pudental SEP and bulbocavernous reflexes (BCR), which are more specific for sacral segments. These modalities allow us to intraoperatively monitor the sacral nervous system (21). But we could not record these modalities which required some special electrodes, because we did not have them and had some technical drawbacks and could not ensure their reliabilities. Hereby, Multimodal IONM is recommended by the International Society of Intraoperative Neurophysiology (ISIN) (2006) for all spinal surgical procedures that have a potential risk of neural structures damage (18).

\section{- CONCLUSION}

Specifically, Multimodal IONM can be recommended for the following spinal pathologies: corrections of spinal deformities with scoliosis greater than 45 degrees, corrections of congenital spine anomalies, resections of intramedullary and extramedullary tumours, extensive anterior and/or posterior decompressions in spinal stenosis in cervical, thoracic and lumbar spine causing myelopathies and functional disturbance of cauda equina and/or individual nerve roots. In addition, IONM equipment must have sufficient channels to monitor all the modalities necessary for the type of surgery being performed. Also, critical modalities in IONM should be performed continuously throughout the surgical procedure.

\section{REFERENCES}

1. Campbell PG, Yadla S, Malone J, Zussman B, Maltenfort MG, Sharan AD, Harrop JS, Ratliff JK: Early complications related to approach in cervical spine surgery: Single-center prospective study. World Neurosurg 74:363-368, 2010

2. Feng B, Qiu G, Shen J, Zhang J, Tian Y, Li S, Zhao H, Zhao Y: Impact of multimodal intraoperative monitoring during surgery for spine deformity and potential risk factors for neurological monitoring changes. J Spinal Disord Tech 25:E108-114, 2012

3. Ghobrial GM, Williams KA Jr, Arnold P, Fehlings M, Harrop JS: latrogenic neurologic deficit after lumbar spine surgery: A review. Clin Neurol Neurosurg 139:76-80, 2015

4. Jankowska E, Padel Y, Tanaka R: Projections of pyramidal tract cells to alpha-motoneurones innervating hind-limb muscles in the monkey. J Physiol 249:637-667, 1975

5. Journee HL, Polak HE, de Kleuver M, Langeloo DD, Postma AA: Improved neuromonitoring during spinal surgery using double-train transcranial electrical stimulation. Med Biol Eng Comput 42:110-113, 2004

6. Journee HL, Polak HE, de Kleuver M: Conditioning stimulation techniques for enhancement of transcranially elicited evoked motor responses. Neurophysiol Clin 37:423-430, 2007

7. Kundnani VK, Zhu L, Tak H, Wong H: Multimodal intraoperative neuromonitoring in corrective surgery for adolescent idiopathic scoliosis: Evaluation of 354 consecutive cases. Indian J Orthop 44:64-72, 2010

8. Langeloo DD, Lelivelt A, Louis JH, Slappendel R, de Kleuver $M$ : Transcranial electrical motor-evoked potential monitoring during surgery for spinal deformity: A study of 145 patients. Spine (Phila Pa 1976) 28:1043-1050, 2003

9. Macdonald DB, Skinner S, Shils J, Yingling C; American Society of Neurophysiological Monitoring: Intraoperative motor evoked potential monitoring -a position statement by the American Society of Neurophysiological Monitoring. Clin Neurophysiol 124(12):2291-2316, 2013

10. Malcharek MJ, Loeffler S, Schiefer D, Manceur MA, Sablotzki A, Gille J, Pilge S, Schneider G: Transcranial motor evoked potentials during anesthesia with desflurane versus propofol-A prospective randomized trial. Clin Neurophysiol 126(9):18251832, 2015

11. McLaren AC, Bailey SI: Cauda equina syndrome: A complication of lumbar discectomy. Clin Orthop Relat Res 204:143-149, 1986

12. Mills KR: The basics of electromyography. J Neurol Neurosurg Psychiatry 76 Suppl 2:ii32-35, 2005

13. Nuwer MR, Emerson RG, Galloway G, Legatt AD, Lopez J, Minahan R, Yamada T, Goodin DS, Armon C, Chaudhry V, Gronseth GS, Harden CL; Therapeutics and Technology Assessment Subcommittee of the American Academy of Neurology; American Clinical Neurophysiology Society: Evidence-based guideline update: Intraoperative spinal monitoring with somatosensory and transcranial electrical motor evoked potentials: Report of the Therapeutics and Technology Assessment Subcommittee of the American Academy of Neurology and the American Clinical Neurophysiology Society. Neurology 78:585-589, 2012 
14. Ramirez LF, Thisted R: Complications and demographic characteristics of patients undergoing lumbar discectomy in community hospitals. Neurosurgery 25:226-230, 1989

15. Recommended standards for neurophysiologic intraoperative monitoring - principles. American Clinical Neurophysiology Society: Guideline 11a. Available at: http://www.acns.org/ $\mathrm{pdfs} / 11 \mathrm{~A}$

16. Sala F, Kothbauer KF: Intraoperative neurophysiology monitoring during surgery for intramedullary spinal cord tumors. In: Nuwer MR (ed), Intraoperative monitoring of neural function. Amsterdam:Elsevier, 2008: 632-650

17. Sala F, Krzan MJ, Deletis V: Intraoperative neurophysiological monitoring in pediatric neurosurgery: Why, when, how? Childs Nerv Syst 18:264-287, 2002

18. Sutter M, Deletis V, Dvorak J, Eggspuehler A, Grob D, Macdonald D, Mueller A, Sala F, Tamaki T: Current opinions and recommendations on multimodal intraoperative monitoring during spine surgeries. Eur Spine J 16 Suppl 2: S232-S237, 2007
19. Tamkus AA, Rice KS, Kim HL: Differential rates of falsepositive findings in transcranial electric motor evoked potential monitoring when using inhalational anesthesia versus total intravenous anesthesia during spine surgeries. Spine J 14(8):1440-1446, 2014

20. Taniguchi M, Cedzich C, Schramm J: Modification of cortical stimulation for motor evoked potentials under general anesthesia: Technical description. Neurosurgery 32: 219-226, 1993

21. Vodusek DB, Deletis V: Intraoperative neurophysiological monitoring of the sacral nervous system. In: Neurophysiology in Neurosurgery, a Modern Intraoperative Approach. USA: Academic Press, 2002: 204-217

22. von Koch CS, Quinones-Hinojosa A, Gulati M, Lyon R, Peacock WJ, Yingling CD: Clinical outcome in children undergoing tethered cord release utilizing intraoperative neurophysiological monitoring. Pediatr Neurosurg 37:81-86, 2002

23. Wang AC, Than KD, Etame AB, La Marca F, Park P: Impact of anesthesia on transcranial electric motor evoked potential monitoring during spine surgery: A review of the literature. Neurosurg Focus 27(4):E7, 2009 